\title{
Thermoelastic noise and homogeneous thermal noise in finite sized gravitational-wave test masses
}

\author{
Yuk Tung Liu and Kip S. Thorne \\ Theoretical Astrophysics, California Institute of Technology, Pasadena, California 91125
}

(Received 16 February 2000; published 20 November 2000)

\begin{abstract}
An analysis is given of thermoelastic noise (thermal noise due to thermoelastic dissipation) in finite sized test masses of laser interferometer gravitational-wave detectors. Finite-size effects increase the thermoelastic noise by a modest amount; for example, for the sapphire test masses tentatively planned for LIGO-II and plausible beam-spot radii, the increase is $\$ 10$ percent. As a side issue, errors are pointed out in the currently used formulas for conventional, homogeneous thermal noise (noise associated with dissipation which is homogeneous and described by an imaginary part of the Young's modulus) in finite sized test masses. Correction of these errors increases the homogeneous thermal noise by $\$ 5$ percent for LIGO-II-type configurations.

PACS number(s): 04.80.Nn, 05.40.-a
\end{abstract}

\section{INTRODUCTION AND SUMMARY}

Internal thermal noise is one of the most dangerous noise sources in a laser interferometer gravitational wave detector in the frequency range $\sim 10 \mathrm{~Hz}$ to $\sim 200 \mathrm{~Hz}$. It is caused by a fluctuational redistribution of thermal energy inside each of the detector's mirror-endowed test masses. This energy redistribution produces a fluctuational change of the test mass's shape and thence a change of the position of its mirrored face, which in turn mimics a gravity-wave-induced motion of the test mass's center of mass [1].

The fluctuation-dissipation theorem [2] describes a relationship between thermal noise and the energy dissipation (entropy increase) that occurs inside the test mass, when the front of the test mass is subjected to an oscillatory driving force [Eq. (3) below]. There are various types of internal thermal noise, each one associated with a specific dissipation mechanism. Until recently, gravitational-wave experimenters have focused almost exclusively on homogeneous thermal noise [1] - i.e., noise associated with all forms of dissipation that are describable by an imaginary part of the Young's modulus which is homogeneous inside the test mass (e.g., dissipation due to homogeneously distributed impurities and dislocations). Thermoelastic dissipation (dissipation due to heat flow down temperature gradients, which are produced by inhomogeneous compression and expansion of the testmass material) is not homogeneous; but until recently it was thought that thermoelastic noise (thermal noise associated with thermoelastic dissipation) would be negligible in LaserInterferometer Gravitational Wave Observatory (LIGO) test masses, compared to homogeneous thermal noise.

Indeed, this is so in the fused silica test masses of LIGO-I detectors-i.e. of the first detectors that will operate in LIGO [3]. However, a careful analysis late last year by Braginsky, Gorodetsky and Vyatchanin [4] (BGV) showed rather convincingly that for the sapphire test masses currently planned for LIGO-II (the second generation detectors in LIGO), thermoelastic noise will be significantly larger than homogeneous thermal noise, and in fact will be so large as to significantly constrain the performance of LIGO-II detectors in the frequency band between $\sim 10 \mathrm{~Hz}$ and $\sim 200 \mathrm{~Hz}$.

The BGV computation of thermoelastic noise was based on an idealization in which each test mass has an arbitrarily large radius and length compared to the size of the light's beam spot on the mirrored test-mass face. In this limiting case, BGV showed that the spectral density $S_{h}(f)$ of the thermoelastic gravitational-wave noise scales as the inverse cube of the beam-spot radius $r_{o}, S_{h} \propto 1 / r_{o}^{3}$, so it is desirable to make $r_{o}$ large. However, when $r_{o}$ is no longer small compared to the test-mass size, the BGV analysis breaks down.

The principal purpose of this paper is to explore, quantitatively, the sign and magnitude of that breakdown. As we shall see, that breakdown (i.e., finite size of the test masses) increases the thermoelastic noise; but for expected beamspot radii $\left(r_{0} \lesssim 3 / 10\right.$ the test-mass radius $a$ ), the increase is modest ( $\lesssim 10$ percent).

A second purpose of this paper is to show how the BGV analysis of thermoelastic noise can be simplified considerably; and [adapting techniques due to Bondu, Hello and Vinet [5] (BHV)], to show how to generalize the BGV analysis to finite sized test masses.

A third purpose is to point out and correct errors in the BHV formulas for homogeneous thermal noise in finite sized test masses (formulas that are currently used in designing test masses and predicting the performance of gravitational wave detectors). The corrections of the BHV formulas increase homogeneous thermal noise by $\$ 5$ percent for beam-spot radii $\lesssim 3 / 10$ the test-mass radius $a$, and thus are primarily of conceptual importance, not practical importance.

In Sec. II, we outline our method of computing thermoelastic noise, in Sec. III we use our method to verify the $\mathrm{BGV}$ result for thermoelastic noise in the limit of arbitrarily large test masses, in Sec. IV we compute the thermoelastic noise in finite sized test masses and estimate the accuracy of our analysis, in Sec. V we correct the errors in the BHV computation of conventional, homogeneous thermal noise, and in Sec. VI we make some concluding remarks.

\section{METHOD OF CALCULATION}

Our analysis of thermoelastic noise is a simplification of one of the procedures developed by BGV: Appendix $\mathrm{C}$ of Ref. [4]. The foundation of the analysis is Levin's [6] " $\mathrm{di}$ rect' method of computing thermal noise (of which ther- 
moelastic noise is a special case):

Levin begins by noting that the gravitational-wave detector's laser beam reads out a difference of generalized positions $q(t)$ of the detector's four test masses, with each $q$ given by an average, over the beam spot's Gaussian power profile, of the normal displacement $\delta z \equiv u_{z}$ of the test-mass face:

$$
\begin{aligned}
q & =\int_{0}^{a} \int_{0}^{2 \pi} \frac{e^{-r^{2} / r_{o}^{2}}}{\pi r_{o}^{2}\left(1-e^{-a^{2} / r_{o}^{2}}\right)} \delta z(r, \phi) r d \phi d r \\
& \simeq \int_{0}^{a} \int_{0}^{2 \pi} \frac{e^{-r^{2} / r_{o}^{2}}}{\pi r_{o}^{2}} \delta z(r, \phi) r d \phi d r .
\end{aligned}
$$

Here $(r, \phi)$ are circular polar coordinates centered on the beam-spot center (which we presume to be at the center of the circular test-mass face), $r_{o}$ is the radius at which the spot's power flux has dropped to $1 / e$ of its central value, and $a$ is the test-mass radius. (The factor $e^{-a^{2} / r_{o}^{2}}$ must be $\ll 1$ in order to keep diffraction losses small, so we shall approximate $1-e^{-a^{2} / r_{o}^{2}}$ by unity throughout this paper.) Levin then appeals to a very general formulation of the fluctuationdissipation theorem, due to Callan and Welton [2], to show that the test-mass thermal noise can be computed by the following thought experiment:

We imagine applying a sinusoidally oscillating pressure

$$
P=F_{o} \frac{e^{-r^{2} / r_{o}^{2}}}{\pi r_{o}^{2}} \cos (\omega t)
$$

to one face of the test mass. Here $F_{o}$ is a constant force amplitude, $\omega=2 \pi f$ is the angular frequency at which one wants to know the spectral density of thermal noise, and the pressure distribution (2) has precisely the same spatial profile as that of the generalized coordinate $q$, whose thermal noise $S_{q}(f)$ one wishes to compute.

The oscillating pressure $P$ feeds energy into the test mass, where it gets dissipated by thermoelastic heat flow. We compute the rate of this energy dissipation, $W_{\text {diss }}$, averaged over the period $2 \pi / \omega$ of the pressure oscillations. ${ }^{1}$ Then the fluctuation-dissipation theorem states that the spectral density of the noise $S_{q}(f)$ is given by

$$
S_{q}(f)=\frac{8 k_{\mathrm{B}} T W_{\mathrm{diss}}}{F_{o}^{2} \omega^{2}}
$$

[Eq. (2) of Ref. [6]]; here $k_{\mathrm{B}}$ is Boltzmann's constant. The interferometer's gravitational-wave signal $h(t)$ is the difference of the generalized positions $q$ of the four test masses, divided by the interferometer arm length $L$. Correspondingly

\footnotetext{
${ }^{1}$ It is here that our analysis is simpler than that of BGV. Instead of computing $W_{\text {diss }}$ and using Eq. (3) for the thermal noise, BGV compute the imaginary part $\Im(\chi)$ of the test-mass susceptibility $\chi$ (which is much harder to compute than $W_{\text {diss }}$ ) and then evaluate $S_{q}$ in terms of $\Im(\chi)$ [their Eq. (14)].
}

the contribution of the test-mass thermoelastic noise to the gravitational-wave noise is $1 / L^{2}$ times the sum of $S_{q}(f)$ over the four test masses (which might have different beam-spot sizes and thus different noises):

$$
S_{h}(f)=\sum_{A=1}^{4} \frac{S_{q_{A}}(f)}{L^{2}} .
$$

The rate $W_{\text {diss }}$ of thermoelastic dissipation is given by the following standard expression [first term of Eq. (35.1) of Landau and Lifshitz [7], cited henceforth as LL]:

$$
W_{\mathrm{diss}}=\left\langle\frac{T d S}{d t}\right\rangle=\left\langle\int \frac{\kappa}{T}(\vec{\nabla} \delta T)^{2} r d \phi d r d z\right\rangle .
$$

Here the integral is over the entire test-mass interior using cylindrical coordinates; $T$ is the unperturbed temperature of the test-mass material and $\delta T$ is the temperature perturbation produced by the oscillating pressure; $d S / d t$ is the rate of increase of the test mass's entropy due to the flux of heat $-\kappa \vec{\nabla} \delta T$ flowing down the temperature gradient $\vec{\nabla} \delta T, \kappa$ is the material's coefficient of thermal conductivity, and $\langle\cdots\rangle$ denotes an average over the pressure's oscillation period $1 / f=2 \pi / \omega$. (For conceptual clarity we explicitly write the average $\langle\cdots\rangle$ throughout this paper, even though in practice it gives just a simple factor $\left\langle\cos ^{2} \omega t\right\rangle=1 / 2$.)

To compute the thermal noise, then, we must calculate the oscillating temperature perturbation $\delta T(r, \phi, z, t)$ inside the test mass, perform the integral (5) over the test-mass interior and the time average to obtain the dissipation rate, then plug that rate into Eq. (3) and then Eq. (4).

The computation of the oscillating temperature perturbation is made fairly simple by two well-justified approximations [4]:

First: The radius and length of the test mass are $a \sim H$ $\sim 14 \mathrm{~cm}$ and the speeds of sound in the test-mass material are $c_{s} \sim 5 \mathrm{~km} / \mathrm{s}$, so the time required for sound to travel across the test mass is $\tau_{\text {sound }} \sim 30 \mu \mathrm{s}$, which is $\sim 300$ times shorter than the gravitational-wave (and pressure-oscillation) period $\tau_{\text {gw }}=1 / f \sim 0.01$ s. This $\tau_{\text {sound }} \ll \tau_{\text {gw }}$ means that we can approximate the oscillations of stress and strain in the test mass, induced by the oscillating pressure $P$, as quasistatic. It seems reasonable to expect this approximation to produce a fractional error

$$
\varepsilon_{\text {quasistatic }} \lesssim \frac{\tau_{\text {sound }}}{\tau_{\text {gw }}}=\frac{f}{f_{\text {sound }}} \sim \frac{1}{300}
$$

in our final answer for the thermoelastic noise $S_{q}(f)$. Here

$$
f_{\text {sound }}=\frac{1}{\tau_{\text {sound }}} \sim \frac{c_{s}}{\min (a, H)} \simeq 30000 \mathrm{~Hz}
$$

for the currently contemplated LIGO-II test masses: sapphire with $a \sim H \sim 14 \mathrm{~cm}$.

Second: The time scale for diffusive heat flow to alter the temperature distribution, $\tau_{T} \sim C_{V} \rho r_{o}^{2} / \kappa \sim 100 \mathrm{~s}$, is $\sim 10^{4}$ times longer than the pressure-oscillation period $\tau_{\mathrm{gw}}$ (here $C_{V} \simeq 7.9 \times 10^{6} \mathrm{erg}^{-1} \mathrm{~K}^{-1}$ is the specific heat per unit 
mass at constant volume, $\rho \simeq 4.0 \mathrm{~g} / \mathrm{cm}^{3}$ is the density, $r_{o}$ $\sim 4 \mathrm{~cm}$ is the spot size and $\kappa$ $\simeq 4.0 \times 10^{6} \mathrm{erg} \mathrm{cm}^{-1} \mathrm{~s}^{-1} \mathrm{~K}^{-1}$ is the thermal conductivity, and our values are for a sapphire test mass). This $\tau_{T}$ $\gg \tau_{\mathrm{gw}}$ means that, when computing the oscillating temperature distribution, we can approximate the oscillations of stress, strain and temperature as adiabatic (negligible diffusive heat flow). The only place that heat flow must be considered is in the volume integral (5) for the dissipation. The dominant contribution to that volume integral will come from a region with radius $\sim r_{o}$ and thickness $\sim r_{o}$ near the beam spot. The region of the integral in which the adiabatic approximation breaks down is predominantly a thin "boundary layer" near the beam spot with radius $r_{o}$ and thickness of order the distance that substantial heat can flow in a time $\sim \tau_{\mathrm{gw}}=1 / f$, i.e., thickness of order

$$
r_{\text {heat }}=\sqrt{\frac{\kappa}{C_{V} \rho f}} \simeq 0.4 \mathrm{~mm} \sqrt{\frac{100 \mathrm{~Hz}}{f}} \text { for sapphire. }
$$

This region of adiabatic breakdown is a fraction $\sim r_{\text {heat }} / r_{o}$ of the region that contributes substantially to the integral, so we expect a fractional error

$$
\varepsilon_{\text {adiabatic }} \sim \frac{r_{\text {heat }}}{r_{o}} \sim 0.01
$$

in $S_{q}(f)$ due to breakdown of the adiabatic approximation.

The quasistatic approximation permits us, at any moment of time $t$, to compute the test mass's internal displacement field $\vec{u}$, and most importantly its expansion

$$
\Theta=\vec{\nabla} \cdot \vec{u}
$$

from the equations of static stress balance [Eq. (7.4) of LL $[7]]$

$$
(1-2 \sigma) \nabla^{2} \vec{u}+\vec{\nabla}(\vec{\nabla} \cdot \vec{u})=0
$$

(where $\sigma$ is the Poisson ratio), with the boundary condition that the normal pressure on the test-mass face be $P(r, t)[\mathrm{Eq}$. (2)] and that all other non-tangential stresses vanish at the test-mass surface. Once $\Theta$ has been computed, we can evaluate the temperature perturbation $\delta T$ from the law of adiabatic temperature change [Eq. (6.5) of LL [7]]

$$
\delta T=\frac{-\alpha_{l} E T}{C_{V} \rho(1-2 \sigma)} \Theta
$$

here $\alpha_{l}$ is the linear thermal expansion coefficient, $E$ is Young's modulus and $C_{V}$ is the specific heat per unit mass at constant volume. ${ }^{2}$ This temperature perturbation can then be plugged into Eq. (5) to obtain the dissipation $W_{\text {diss }}$ as an integral over the gradient of the expansion

\footnotetext{
${ }^{2} \mathrm{LL}$ use the volumetric thermal expansion coefficient $\alpha=3 \alpha_{l}$ and the specific heat per unit volume $C_{v}=\rho C_{V}$.
}

$$
\left.W_{\mathrm{diss}}=\kappa T\left(\frac{E \alpha_{l}}{(1-2 \sigma) C_{V} \rho}\right)^{2} / \int(\vec{\nabla} \Theta)^{2} r d \phi d r d z\right) .
$$

This $W_{\text {diss }}$ can be inserted into Eq. (3) to obtain the thermoelastic noise.

\section{INFINITE TEST MASSES}

\section{A. Dissipation and noise computed via BGV techniques}

We illustrate the above computational procedure by using it to verify the BGV [4] result for thermoelastic noise in the case where each test mass is arbitrarily large compared to the spot size.

Following BGV, we approximate the test mass as an infinite half space. Then the solution to the quasistatic stressbalance equation (11) is given by a Green's-function expression $\left[\mathrm{LL}\right.$ Eq. (8.18) with $\left.F_{x}=F_{y}=0, F_{z}=P(r, \phi)\right]$, integrated over the surface of the test mass. Taking the divergence of that expression [or, equivalently, taking the divergence of Eq. (39) of BGV], we obtain the following equation for the pressure-induced expansion:

$$
\begin{aligned}
\Theta= & -\frac{(1+\sigma)(1-2 \sigma) F_{o}}{\pi^{2} r_{o}^{2} E} \cos (\omega t) \\
& \times z \iint_{-\infty}^{+\infty} d x^{\prime} d y^{\prime} \frac{e^{-\left(x^{\prime 2}+y^{\prime 2}\right) / r_{o}^{2}}}{\left[\left(x-x^{\prime}\right)^{2}+\left(y-y^{\prime}\right)^{2}+z^{2}\right]^{3 / 2}},
\end{aligned}
$$

where we have converted from polar coordinates to Cartesian coordinates. Following a clever procedure implicit in the $\mathrm{BGV}$ analysis [in going from their Eq. (39) to (40)], we insert into the integral (14) an integral of the Dirac delta function written as

$$
\int_{-\infty}^{+\infty} \delta\left(x-x^{\prime}-x^{\prime \prime}\right) d x^{\prime \prime}=\frac{1}{2 \pi} \iint_{-\infty}^{+\infty} e^{i k_{x}\left(x-x^{\prime}-x^{\prime \prime}\right)} d k_{x} d x^{\prime \prime}
$$

and a similar expression for $\int \delta\left(y-y^{\prime}-y^{\prime \prime}\right) d y^{\prime \prime}$, and we rewrite $x-x^{\prime}$ and $y-y^{\prime}$ in the denominator as $x^{\prime \prime}$ and $y^{\prime \prime}$, thereby obtaining a new version of Eq. (14) with integrals over $k_{x}, k_{y}, x^{\prime}, y^{\prime}, x^{\prime \prime}, y^{\prime \prime}$. The integrals over $x^{\prime}, y^{\prime}, x^{\prime \prime}, y^{\prime \prime}$ are then readily carried out analytically (they are well-known Fourier transforms), to yield Eq. (40) of BGV: ${ }^{3}$

$$
\begin{aligned}
\Theta= & -\frac{(1+\sigma)(1-2 \sigma) F_{o}}{2 \pi^{2} E} \cos \omega t \\
& \times \iint_{-\infty}^{+\infty} e^{-k_{\perp}^{2} r_{o}^{2} / 4} e^{-k_{\perp} z} e^{i\left(k_{x} x+k_{y} y\right)} d k_{x} d k_{y},
\end{aligned}
$$

\footnotetext{
${ }^{3}$ Note that our notation differs slightly from that of BGV: Our $x$ is their $z$, our $z$ is their $x$, and they have factored out the $\cos \omega t$, which they write as $e^{i \omega t}$.
} 
where $k_{\perp} \equiv \sqrt{k_{x}^{2}+k_{y}^{2}}$.

It is straightforward to take the gradient of this expression, square it (with one term an integral over $k_{x}, k_{y}$ and the other over $k_{x}^{\prime}, k_{y}^{\prime}$ ), and integrate over $x$ and $y$ (from $-\infty$ to $+\infty$ ) and over $z$ (from 0 to $\infty$ ); the result is $\int(\vec{\nabla} \Theta)^{2} d x d y d z$ expressed as an integral over $x, y, z, k_{x}, k_{y}, k_{x}^{\prime}, k_{y}^{\prime}$. The integrals can be done easily, first over $z$ to get $1 /\left(k_{\perp}+k_{\perp}^{\prime}\right)$, then over $x$ and $y$ to get Dirac delta functions, then over the $k$ 's. The result, when inserted into Eq. (13), is

$$
W_{\mathrm{diss}}=\frac{(1+\sigma)^{2} \kappa \alpha_{l}^{2} T}{\sqrt{2 \pi} C_{V}^{2} \rho^{2} r_{o}^{3}} F_{o}^{2} .
$$

By then inserting this into Eq. (3), we obtain the BGV result for the thermoelastic noise [their Eq. (12)]

$$
S_{q}^{\mathrm{ITM}}(f)=\frac{8(1+\sigma)^{2} \kappa \alpha_{l}^{2} k_{\mathrm{B}} T^{2}}{\sqrt{2 \pi} C_{V}^{2} \rho^{2} r_{o}^{3} \omega^{2}} .
$$

Here the superscript ITM means for an 'infinite test mass.'

\section{B. Derivation via BHV techniques}

Equation (17) for $W_{\text {diss }}$ can also be derived in cylindrical coordinates $(r, z, \phi)$ using the techniques of BHV [5]: The displacement $\vec{u}$ has components [BHV Eqs. (5) and (6) with the denominator in Eq. (5) corrected from $\mu$ to $\mu+\lambda$ and with $\beta=\alpha$; see passage following BHV Eq. (8)]

$$
\begin{aligned}
& u_{r}=\int_{0}^{\infty} \alpha(k)\left(1-\frac{\lambda+2 \mu}{\lambda+\mu}+k z\right) e^{-k z} J_{1}(k r) k d k, \\
& u_{z}=\int_{0}^{\infty} \alpha(k)\left(1+\frac{\mu}{\lambda+\mu}+k z\right) e^{-k z} J_{0}(k r) k d k, \\
& u_{\phi}=0
\end{aligned}
$$

where

$$
\alpha(k)=\frac{e^{-k^{2} r_{o}^{2} / 4}}{4 \pi \mu k} F_{o} \cos \omega t
$$

[BHV Eq. (11), with the overall sign corrected from - to + , with $w_{o}=\sqrt{2} r_{o}$ cf. BHV Eq. (2), and with $F_{o} \cos \omega t$ inserted because our method of applying the fluctuation-dissipation theorem is dynamical while BHV's method is static and BHV set $\left.F_{o}=1\right]$. In Eqs. (19) the $J_{n}$ are Bessel functions and $\lambda$ and $\mu$ are the Lamé coefficients (and $\mu$ is also the shear modulus), which are related to the Young's modulus $E$ and the Poisson ratio $\sigma$ by

$$
\lambda=\frac{E \sigma}{(1-2 \sigma)(1+\sigma)}, \quad \mu=\frac{E}{2(1+\sigma)} .
$$

The divergence of the displacement (19) is

$$
\Theta=-\frac{2 \mu}{\lambda+\mu} \int_{0}^{\infty} \alpha(k) e^{-k z} J_{0}(k r) k^{2} d k
$$

The nonzero components of the gradient of this expansion are

$$
\begin{aligned}
& \frac{\partial \Theta}{\partial r}=\frac{2 \mu}{\lambda+\mu} \int_{0}^{\infty} \alpha(k) e^{-k z} J_{1}(k r) k^{3} d k, \\
& \frac{\partial \Theta}{\partial z}=\frac{2 \mu}{\lambda+\mu} \int_{0}^{\infty} \alpha(k) e^{-k z} J_{0}(k r) k^{3} d k .
\end{aligned}
$$

By squaring the gradient, integrating over the interior of the test mass, and using the relations

$$
\int_{0}^{\infty} J_{n}(k r) J_{n}\left(k^{\prime} r\right) r d r=\frac{\delta\left(k-k^{\prime}\right)}{k}
$$

(which follow from the Fourier-Bessel integral), and by replacing the Lamé coefficients by the Poisson ratio and Young's modulus [Eqs. (21)], and inserting the resulting $\int(\vec{\nabla} \Theta)^{2} r d \phi d r d z$ into expression (13), we obtain the same result (17) as we got using BGV techniques. By inserting this into Eq. (13), we obtain the thermoelastic noise (18).

\section{FINITE SIZED TEST MASSES}

\section{A. BHV solution for displacement}

Consider a finite sized, cylindrical test mass with radius $a$ and thickness $H$, and with the Gaussian shaped light spot centered on the cylinder's circular face. For this case, Bondu, Hello and Vinet (BHV) [5] have constructed a rather accurate but approximate solution of the static elasticity equations. Unfortunately, their solution satisfies the wrong boundary conditions and thus must be corrected:

The error arises when BHV expand the Gaussian-shaped pressure (2) as a sum over Bessel functions. BHV incorrectly omit a uniform-pressure term from the sum. As a result, the pressure that they imagine applying to the test-mass face [their Eq. (18)],

$$
P_{\mathrm{BHV}}(r)=F_{o} \cos \omega t \sum_{m=1}^{\infty} p_{m} J_{0}\left(k_{m} r\right)
$$

[where $J_{0}$ is the Bessel function of order zero, $k_{m}$ is related to the $m$ 'th zero $\zeta_{m}$ of the order-one Bessel function $J_{1}(x)$ by $k_{m}=\zeta_{m} / a$, and $p_{m}$ are constant coefficients given below], has a vanishing surface integral

$$
\int_{0}^{a} P_{\mathrm{BHV}} 2 \pi r d r=0
$$

In other words, their applied pressure (25) is equal to the desired pressure $P(r)$ [Eq. (2)] minus an equal and opposite net force $F_{o} \cos (\omega t)$ applied uniformly over the test-mass face:

$$
\begin{aligned}
P_{\mathrm{BHV}}(r) & =P(r)-p_{0} F_{o} \cos \omega t ; \\
p_{0} & \equiv \frac{1}{\pi a^{2}} .
\end{aligned}
$$


[Recall that we are approximating $1-e^{-a^{2} / r_{0}^{2}}$ by unity; see discussion following Eq. (1).]

It is evident, then, that to get the correct distribution of elastic displacement $\vec{u}$ inside the test mass, we must add to the BHV displacement a correction. This correction is the displacement caused by the spatially uniform pressure $p_{0} F_{o} \cos \omega t$ on the test-mass face. That uniform pressure causes the test mass to accelerate with acceleration $\vec{a}$ $=\left[\left(F_{o} \cos \omega t\right) / M\right] \vec{e}_{z}$, where $M=\pi a^{2} H \rho$ is the mass of the test mass and $\rho$ is its density. In the reference frame of the accelerating test mass, all parts of the test mass feel a "gravitational" acceleration $g \vec{e}_{z}$ equal and opposite to $\vec{a}$, i.e. $g=$ $-\left(F_{o} \cos \omega t\right) / M$ (which can be treated as quasistatic, though it oscillates at frequency $\omega$ ). Thus, the displacement is the same as would occur if the test mass were to reside in the gravitational field $g \vec{e}_{z}$ with a uniform pressure on its face counteracting the force of gravity. The solution for this displacement is given by LL [7] (problem 1, p. 18). ${ }^{4}$ Translating into our notation and converting from the Young's modulus and Poisson ratio to the Lamé coefficients via Eq. (21), we obtain

$\frac{\delta u_{r}}{F_{o} \cos \omega t}=\frac{\lambda p_{0} r}{2 \mu(3 \lambda+2 \mu)}\left(1-\frac{z}{H}\right)$,

$\frac{\delta u_{z}}{F_{o} \cos \omega t}=\frac{\lambda p_{0} r^{2}}{4 \mu H(3 \lambda+2 \mu)}-\frac{(\lambda+\mu) p_{0}}{\mu(3 \lambda+2 \mu)}\left(z-\frac{z^{2}}{2 H}\right)$.

The total corrected displacement, in cylindrical coordinates, is

$$
u_{r}=u_{r}^{\mathrm{BHV}}+\delta u_{r}, \quad u_{z}=u_{z}^{\mathrm{BHV}}+\delta u_{z}, \quad u_{\phi}=0,
$$

where $u_{j}^{\mathrm{BHV}}$ is the BHV displacement [their Eqs. (15) plus (25) and (17) plus (26)]:

$$
\begin{aligned}
\frac{u_{r}^{\mathrm{BHV}}(r, z)}{F_{o} \cos \omega t}= & \frac{\lambda+2 \mu}{2 \mu(3 \lambda+2 \mu)}\left(c_{0} r+c_{1} r z\right) \\
& +\sum_{m=1}^{\infty} A_{m}(z) J_{1}\left(k_{m} r\right),
\end{aligned}
$$

\footnotetext{
${ }^{4} \mathrm{LL}$ seek to solve a problem in which (in the presence of the uniform gravitational acceleration), instead of having a uniform pressure applied to the face of the cylindrical test mass, the face has vanishing displacement. Their solution actually satisfies our desired boundary conditions but not theirs; therefore, they comment on it being inaccurate near the test-mass face. For our problem it is accurate.
}

$$
\begin{aligned}
\frac{u_{z}^{\mathrm{BHV}}(r, z)}{F_{o} \cos \omega t}= & -\frac{\lambda}{\mu(3 \lambda+2 \mu)}\left(c_{0} z+\frac{c_{1} z^{2}}{2}\right) \\
& -\frac{\lambda+2 \mu}{4 \mu(3 \lambda+2 \mu)} c_{1} r^{2} \\
& +\sum_{m=1}^{\infty} B_{m}(z) J_{0}\left(k_{m} r\right), \\
u_{\phi}^{\mathrm{BHV}}(r, z)= & 0 .
\end{aligned}
$$

Here the coefficients $c_{0}$ and $c_{1}$ are [equations following Eqs. (24) and (26) of BHV]

$$
c_{0}=6 \frac{a^{2}}{H^{2}} \sum_{m=1}^{\infty} \frac{J_{0}\left(\zeta_{m}\right) p_{m}}{\zeta_{m}^{2}}, \quad c_{1}=\frac{-2 c_{0}}{H}
$$

and $A_{m}$ and $B_{m}$ are the following functions of $z$ [Eqs. (19) and (20) of BHV]:

$$
\begin{aligned}
A_{m}(z)= & \gamma_{m} e^{-k_{m} z}+\delta_{m} e^{k_{m} z} \\
& +\frac{k_{m} z}{2} \frac{\lambda+\mu}{\lambda+2 \mu}\left(\alpha_{m} e^{-k_{m} z}+\beta_{m} e^{k_{m} z}\right) \\
B_{m}(z)= & {\left[\frac{\lambda+3 \mu}{2(\lambda+2 \mu)} \beta_{m}-\delta_{m}\right] e^{k_{m} z} } \\
& +\left[\frac{\lambda+3 \mu}{2(\lambda+2 \mu)} \alpha_{m}+\gamma_{m}\right] e^{-k_{m} z} \\
& +\frac{k_{m} z}{2} \frac{\lambda+\mu}{\lambda+2 \mu}\left(\alpha_{m} e^{-k_{m} z}-\beta_{m} e^{k_{m} z}\right),
\end{aligned}
$$

where $\alpha_{m}, \beta_{m}, \gamma_{m}$ and $\delta_{m}$ are constants given by [Eqs. (21)-(24) of BHV]:

$Q_{m}=\exp \left(-2 k_{m} H\right)$

$$
\begin{aligned}
& \alpha_{m}=\frac{p_{m}(\lambda+2 \mu)}{k_{m} \mu(\lambda+\mu)} \frac{1-Q_{m}+2 k_{m} H Q_{m}}{\left(1-Q_{m}\right)^{2}-4 k_{m}^{2} H^{2} Q_{m}} \\
& \beta_{m}=\frac{p_{m}(\lambda+2 \mu) Q_{m}}{k_{m} \mu(\lambda+\mu)} \frac{1-Q_{m}+2 k_{m} H}{\left(1-Q_{m}\right)^{2}-4 k_{m}^{2} H^{2} Q_{m}}
\end{aligned}
$$

$$
\begin{aligned}
\gamma_{m}= & -\frac{p_{m}}{2 k_{m} \mu(\lambda+\mu)} \\
& \times \frac{\left[2 k_{m}^{2} H^{2}(\lambda+\mu)+2 \mu k_{m} H\right] Q_{m}+\mu\left(1-Q_{m}\right)}{\left(1-Q_{m}\right)^{2}-4 k_{m}^{2} H^{2} Q_{m}}
\end{aligned}
$$




$$
\begin{aligned}
\delta_{m}= & -\frac{p_{m} Q_{m}}{2 k_{m} \mu(\lambda+\mu)} \\
& \times \frac{2 k_{m}^{2} H^{2}(\lambda+\mu)-2 \mu k_{m} H-\mu\left(1-Q_{m}\right)}{\left(1-Q_{m}\right)^{2}-4 k_{m}^{2} H^{2} Q_{m}},
\end{aligned}
$$

with [equation following Eq. (18) in BHV]

$$
p_{m}=\frac{2}{a^{2} J_{0}^{2}\left(\zeta_{m}\right)} \int_{0}^{a} \frac{e^{-r^{2} / r_{o}^{2}}}{\pi r_{o}^{2}} J_{0}\left(k_{m} r\right) r d r .
$$

In the spirit of our approximating $1-e^{-a^{2} / r_{o}^{2}}$ by unity [discussion following Eq. (1)], BHV suggest approximating the upper limit of this integral by $\infty$; the integral can then be done analytically, giving [equation preceding Eq. (19) of $\mathrm{BHV}]$

$$
p_{m}=\frac{\exp \left(-k_{m}^{2} r_{o}^{2} / 4\right)}{\pi a^{2} J_{0}^{2}\left(\zeta_{m}\right)} .
$$

This is a good approximation to the exact formula (36) for small $m$ (which turn out to give the dominant contribution to the noise), but for large $m$ it can severely underestimate $p_{m}$.

\section{B. Expansion and the integral of its squared gradient}

It is straightforward to compute the expansion $\Theta=\vec{\nabla} \cdot \vec{u}$ and the components of its gradient from expressions (29), (31) and (30); the results are

$$
\begin{aligned}
\frac{\Theta(r, z)}{F_{o} \cos \omega t}= & -\frac{p_{0}}{3 \lambda+2 \mu}\left(1-\frac{z}{H}\right)+\frac{2\left(c_{0}+c_{1} z\right)}{3 \lambda+2 \mu} \\
& +\sum_{m=1}^{\infty}\left[k_{m} A_{m}(z)+B_{m}^{\prime}(z)\right] J_{0}\left(k_{m} r\right),
\end{aligned}
$$

and

$$
\begin{aligned}
\frac{\partial \Theta / \partial r}{F_{o} \cos \omega t}= & -\sum_{m=1}^{\infty} k_{m}\left[k_{m} A_{m}(z)+B_{m}^{\prime}(z)\right] J_{1}\left(k_{m} r\right), \\
\frac{\partial \Theta / \partial z}{F_{o} \cos \omega t}= & \frac{2 \tilde{c}_{1}}{3 \lambda+2 \mu}+\sum_{m=1}^{\infty}\left[k_{m} A_{m}^{\prime}(z)\right. \\
& \left.+B_{m}^{\prime \prime}(z)\right] J_{0}\left(k_{m} r\right),
\end{aligned}
$$

where the primes denote derivatives with respect to $z$ and the coefficient $\tilde{c}_{1}$ is

$$
\tilde{c}_{1}=c_{1}+\frac{p_{0}}{2 H} \text {. }
$$

Using the (nonstandard) orthogonality relations

$$
\int_{0}^{a} r J_{1}\left(k_{m} r\right) J_{1}\left(k_{n} r\right) d r=\frac{a^{2}}{2} J_{0}^{2}\left(\zeta_{m}\right) \delta_{m n},
$$

$$
\begin{gathered}
\int_{0}^{a} r J_{0}\left(k_{m} r\right) J_{0}\left(k_{n} r\right) d r=\frac{a^{2}}{2} J_{0}^{2}\left(\zeta_{m}\right) \delta_{m n}, \\
\int_{0}^{a} r J_{0}\left(k_{m} r\right) d r=0,
\end{gathered}
$$

the volume integral of $(\vec{\nabla} \Theta)^{2}$ can be evaluated analytically. The result, after some algebra and after averaging $\cos ^{2} \omega t$ to $1 / 2$, is

$$
\begin{aligned}
\frac{1}{F_{o}^{2}} \iint & \left.(\vec{\nabla} \Theta)^{2} r d \phi d r d z\right\rangle \\
= & \frac{2 \pi a^{2} \widetilde{c}_{1}^{2} H}{(3 \lambda+2 \mu)^{2}} \\
& +\frac{\pi a^{2}}{2(\lambda+\mu)^{2}} \sum_{m=1}^{\infty} \frac{k_{m} p_{m}^{2}\left(1-Q_{m}\right) J_{0}^{2}\left(\zeta_{m}\right)}{\left[\left(1-Q_{m}\right)^{2}-4 H^{2} k_{m}^{2} Q_{m}\right]^{2}} \\
& \times\left[\left(1-Q_{m}\right)^{2}\left(1+Q_{m}\right)+8 H k_{m} Q_{m}\left(1-Q_{m}\right)\right. \\
& \left.+4 H^{2} k_{m}^{2} Q_{m}\left(1+Q_{m}\right)\right] .
\end{aligned}
$$

\section{Thermoelastic noise}

Inserting Eq. (44) into Eq. (13) and then into Eq. (3), and using Eqs. (21) for the Lamé coefficients, we obtain for the spectral density of thermoelastic noise in a finite sized test mass:

$$
S_{q}^{\mathrm{FTM}}=C_{\mathrm{FTM}}^{2} S_{q}^{\mathrm{ITM}} .
$$

Here $S_{q}^{I T M}$ is the BGV result (18) for the spectral density for an infinite test mass, and $C_{\mathrm{FTM}}^{2}$ is the following finite-testmass correction to the spectral density:

$$
\begin{aligned}
C_{\mathrm{FTM}}^{2}= & \frac{(2 \pi)^{3 / 2} r_{o}^{3}}{a^{3}}\left\{\frac{a^{5} H \widetilde{c}_{1}^{2}}{(1+\sigma)^{2}}\right. \\
& +\sum_{m=1}^{\infty} \frac{a^{5} k_{m} p_{m}^{2}\left(1-Q_{m}\right) J_{0}^{2}\left(\zeta_{m}\right)}{\left[\left(1-Q_{m}\right)^{2}-4 H^{2} k_{m}^{2} Q_{m}\right]^{2}} \\
& \times\left[\left(1-Q_{m}\right)^{2}\left(1+Q_{m}\right)+8 H k_{m} Q_{m}\left(1-Q_{m}\right)\right. \\
& \left.\left.+4 H^{2} k_{m}^{2} Q_{m}\left(1+Q_{m}\right)\right]\right\} .
\end{aligned}
$$

The square root, $C_{\mathrm{FTM}}$, of this finite-test-mass correction is plotted in Fig. 1 as a function of the test-mass thickness $H$ and radius $a$ measured in units of the beam-spot radius $r_{o}$. [One can easily show from Eq. (46) that $C_{\mathrm{FTM}}$ depends on $H, a$ and $r_{o}$ only through the dimensionless ratios $H / r_{o}$ and $a / r_{o}$, as must be the case on dimensional grounds.] Notice that the noise is larger, at fixed $r_{o}$, for large- $a$, small- $H$ test masses (thin disks) than for small- $a$, large- $H$ test masses (long cylinders). However, for plausible parameters the difference is only a few tens of percent. The reason for the 


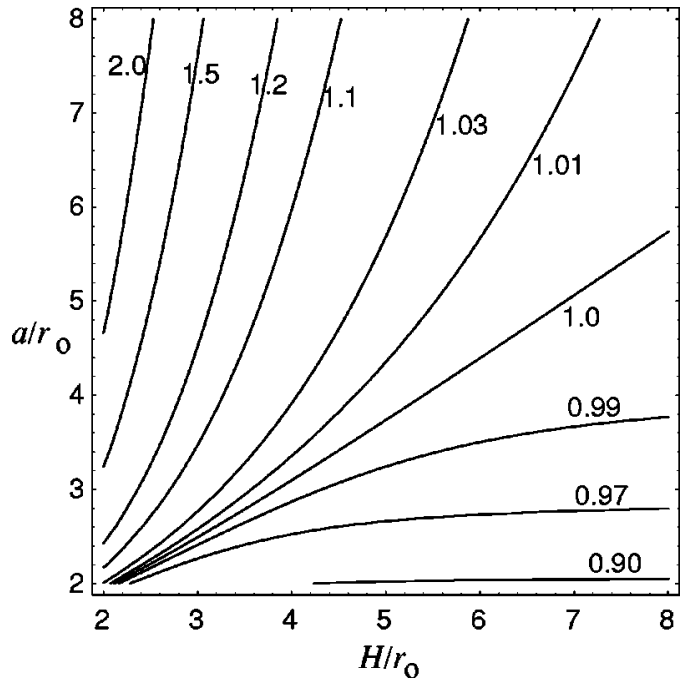

FIG. 1. Contour plot of the correction $C_{\mathrm{FTM}}$ to the thermoelastic amplitude noise $\sqrt{S_{q}(f)}$ due to the finite size of the test mass [Eqs. (45) and (46)]. This correction is shown as a function of the testmass radius $a$ and thickness $H$, both measured in units of the beamspot radius $r_{o}$ (the radius at which the light beam's energy flux has dropped to $1 / e$ of its central value.)

greater noise in a thin disk is that it experiences greater deformation, when a force acts at the center of its face, than does a long cylinder, and thus the integral (13), to which the noise is proportional, is larger. (See, e.g., Sec. 12 of [7], or Sec. 305 of [8].)

The current "straw-man" ("reference") design for LIGO-II includes sapphire test masses with $a=14 \mathrm{~cm}$ and $H=12.2 \mathrm{~cm}$. In Fig. 2 we plot the finite-test-mass correction $C_{\mathrm{FTM}}$ as a function of beam-spot radius $r_{o}$ (in centimeters) for such test masses (for which we use the BGV values of the parameters $\alpha=5.0 \times 10^{-6} \mathrm{~K}^{-1}, \quad \kappa=4.0 \times 10^{6}$ $\operatorname{erg~K} \mathrm{K}^{-1} \mathrm{~cm}^{-1} \mathrm{~s}^{-1}, \rho=4.0 \mathrm{~g} / \mathrm{cm}^{3}, C_{V}=7.9 \times 10^{6} \mathrm{erg}$ $\left.\mathrm{g}^{-1} \mathrm{~K}^{-1}, E=4 \times 10^{12} \mathrm{erg} / \mathrm{cm}^{3}, \sigma=0.29\right)$. Although we continue our plot up to $r_{o}=6 \mathrm{~cm}$, it may be impractical or undesirable to operate with $r_{o}$ much larger than $4 \mathrm{~cm}$. Two reasons for this are: (i) Each time the light beam encounters a test mass, a fraction $\sim e^{-a^{2} / r_{o}^{2}}$ of its power is lost around the test-mass sides ("diffraction losses"); keeping this be-

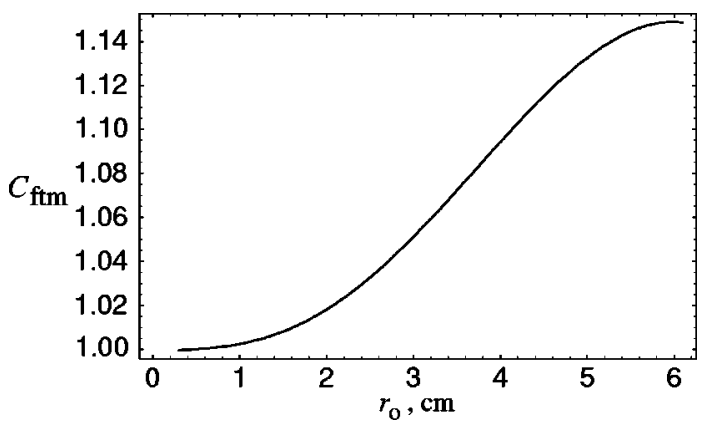

FIG. 2. Correction $C_{\text {FTM }}$ to the thermoelastic amplitude noise as a function of the beam-spot radius $r_{o}$, for test masses with the parameters currently being contemplated for LIGO-II: $30 \mathrm{~kg}$ sapphire cylinders with radius $a=14 \mathrm{~cm}$ and thickness $H=12.2 \mathrm{~cm}$. low $\sim 10 \mathrm{ppm}$ requires $r_{o} \lesssim 4 \mathrm{~cm}$. (ii) There are practical limitations $R \leqq 50 \mathrm{~km}$ on the radii of curvature of the testmass mirrors; if the beam waist is half way between the mirrors of an arm's optical cavity so the spot sizes $r_{o}$ are the same on the two mirrors, and if $R$ is significantly larger than the arm length $L=4 \mathrm{~km}$, then the spot sizes are $r_{o}$ $\simeq\left(\lambda^{2} L R / 8 \pi^{2}\right)^{1 / 4}$ (where $\lambda=1.06 \mu \mathrm{m}$ is the light wavelength), so $R \leqq 50 \mathrm{~km}$ requires $r_{o} \lesssim 4 \mathrm{~cm}$.

For the plausible range $r_{o} \lesssim 4 \mathrm{~cm}$, Fig. 2 shows that the finite-test-mass correction to the amplitude noise is $\lesssim 10$ percent.

\section{Errors in our analysis}

There are three significant sources of error in our analysis. We expect them to produce a net error in $C_{\mathrm{FTM}}$ and thence in the test-mass noise $\sqrt{S_{q}^{\mathrm{FTM}}}$ that is $\lesssim 1$ percent, for the expected LIGO-II parameter regime $(a \sim 14 \mathrm{~cm}, H$ $\left.\sim 12 \mathrm{~cm}, r_{o} \lesssim 4 \mathrm{~cm}\right)$. More specifically:

One error source is the quasistatic approximation. We have already estimated this as producing a fractional error $\varepsilon_{\text {quasistatic }} \leq 0.003$ in $S_{h}$ [Eq. (6)], and the error in $\sqrt{S_{q}}$ will be half this, $\leq 0.0015$.

The second error source is the adiabatic approximation. We have already estimated that this produces a fractional error $\varepsilon_{\text {adiabatic }} \sim 0.01$ in $S_{q}$ [Eq. (9)], and the error in $\sqrt{S_{q}}$ will be half this, $₫ 0.005$.

The third error source is one that we have not discussed: A failure of the elastic displacement (31) to satisfy the boundary condition $T_{r r}=0$ on the test mass's cylindrical sides, $r=a$. As was discussed by BHV [5], the $c_{0}$ and $c_{1}$ terms in the displacement (31) are a correction to the leading-order displacement, designed to improve the satisfaction of the $T_{r r}(a)=0$ boundary condition. We shall refer to these terms as the "Saint-Venant correction" [5]. In our final answer for $S_{q}(f)$ [Eqs. (45) and (46)], this Saint-Venant correction makes a fractional contribution $\lesssim 6$ per cent, for LIGO-II type test masses and plausible beam radii $r_{o}$ $\$ 4 \mathrm{~cm}$. The rms value of $T_{r r}(a)$ with the Saint-Venant correction included is smaller than that without the SaintVenant correction by about a factor 3 , so it is reasonable to expect that the remaining error in $S_{q}(f)$ due to $T_{r r}(a) \neq 0$ is $\leqslant 1 / 3$ of the Saint-Venant correction, i.e., a remaining fractional error

$$
\varepsilon_{\mathrm{SV}} \lesssim \frac{1}{3} \times 0.06=0.02 \text {. }
$$

The fractional error in $\sqrt{S_{q}}$ will be half this, $\leq 0.01$-which is larger than the other two errors.

Combining these three errors in quadrature, we expect our formulas for $\sqrt{S_{q}^{F T M}}$ to make a net fractional error of magnitude

$$
\frac{\delta C_{\mathrm{FTM}}}{C_{\mathrm{FTM}}}=\frac{\delta \sqrt{S_{q}^{\mathrm{FTM}}}}{\sqrt{S_{q}^{\mathrm{FTM}}}} \lesssim 0.01
$$


for LIGO-II-type test masses and beam-spot radii $r_{o}$ $\lesssim 4 \mathrm{~cm}$.

\section{CONVENTIONAL THERMAL NOISE}

Because of the boundary-condition error that BHV make in solving the elasticity equations (and because of an additional algebraic error discussed below), their result for the conventional thermal noise must be corrected.

The conventional thermal noise is given by Levin's formula (3) with $W_{\text {diss }}$ the time-averaged dissipation produced by an imaginary part $\mathfrak{I}(E)=\Phi(\omega) E$ of the Young's modulus:

$$
\begin{aligned}
W_{\text {diss }} & =2 \omega \Phi(\omega)\langle U\rangle \\
& =\omega \Phi(\omega) \int\left\langle\lambda \Theta^{2}+2 \mu S_{i j} S_{i j}\right\rangle r d r d \phi d z .
\end{aligned}
$$

Here $\langle U\rangle$ is the time-averaged elastic energy, $S_{i j} S_{i j}$ is the square of the strain associated with the displacement $\vec{u}$, there is an implied sum over $i$ and $j$, and the integral is over the test-mass interior; cf. Eq. (12) of Ref. [6].

The expansion $\Theta$ is given by Eq. (38), and the components of the strain on the spherical, orthonormal basis $\vec{e}_{r}, \vec{e}_{\phi}, \vec{e}_{z}$ are readily computable from the displacement (30), (29), (31) via [Eqs. (A.1)-(A.4) of BHV]

$$
\begin{aligned}
& S_{r r}=u_{r, r}, \quad S_{\phi \phi}=\frac{u_{r}}{r}, \quad S_{z z}=u_{z, z}, \\
& S_{r z}=S_{z r}=\frac{1}{2}\left(u_{z, r}+u_{r, z}\right),
\end{aligned}
$$

where commas denote partial derivatives. By evaluating these strain components, inserting them and the expansion (38) into Eq. (49), averaging over time, integrating over the test mass, and reexpressing the Lamé coefficients in terms of the Young's modulus and Poisson ratio, we obtain

$$
W_{\mathrm{diss}}=\omega \Phi(\omega)\left(U_{o}+\Delta U\right) F_{o}^{2} .
$$

Here $U_{o}$ is given by

$$
U_{o}=\frac{\left(1-\sigma^{2}\right) \pi a^{3}}{E} \sum_{m=1}^{\infty} U_{m} \frac{p_{m}^{2} J_{o}^{2}\left(\zeta_{m}\right)}{\zeta_{m}},
$$

with [equation following Eq. (29) of BHV]

$$
U_{m}=\frac{1-Q_{m}^{2}+4 k_{m} H Q_{m}}{\left(1-Q_{m}\right)^{2}-4 k_{m}^{2} H^{2} Q_{m}}
$$

while $\Delta U$ is

$$
\Delta U=\frac{a^{2}}{6 \pi H^{3} E}\left[\pi^{2} H^{4} p_{0}^{2}+12 \pi H^{2} \sigma p_{0} s+72(1-\sigma) s^{2}\right],
$$

with

$$
s=\pi a^{2} \sum_{m=1}^{\infty} \frac{p_{m} J_{0}\left(\zeta_{m}\right)}{\zeta_{m}^{2}}
$$

When the approximation (37) is made for $p_{m}, U_{o}$ takes the form given by BHV [their Eq. (30)]

$$
U_{o}=\frac{1-\sigma^{2}}{\pi a E} \sum_{m=1}^{\infty} U_{m} \frac{\exp \left(-\zeta_{m}^{2} r_{o}^{2} / 2 a^{2}\right)}{\zeta_{m} J_{0}\left(\zeta_{m}\right)^{2}}
$$

and $s$ takes the form

$$
s=\sum_{m=1}^{\infty} \frac{\exp \left(-\zeta_{m}^{2} r_{0}^{2} / 4 a^{2}\right)}{\zeta_{m}^{2} J_{0}\left(\zeta_{m}\right)}
$$

The approximations (56) and (57) are rather good for realistic parameter values, despite the fact that for large $m$ Eq. (37) is a very poor approximation to $p_{m}$, because large $m$ make small contributions to $U_{o}$ and $s$.

Equations (54) and (57) for $\Delta U$ differ from Eq. (31) of BHV for two reasons: (i) BHV used the wrong boundary conditions at the test-mass face [see beginning of Sec. IV A above]; correcting this leads to all the terms in Eq. (54) involving $p_{o}$. (ii) BHV seem to have made an algebraic error: Eqs. (54) and (57) should agree with BHV Eq. (31) when $p_{o}$ is set to zero, but they do not; it might be that BHV accidentally omitted the $S_{\phi \phi}^{2}$ term or the $S_{r r}^{2}$ term when evaluating Eq. (49).

Inserting Eq. (51) into Eq. (3), we obtain the BHV expression for the conventional thermal noise [their equation following Eq. (31)]

$$
S_{q}^{\mathrm{FTM}}(f)=\frac{8 k_{\mathrm{B}} T}{\omega} \Phi(\omega)\left(U_{o}+\Delta U\right),
$$

where (to reiterate) $U_{o}$ is given by Eqs. (52) [or (56)] and (53), while $\Delta U$ is given by Eqs. (54) and (55) [or (57)].

If the test mass is infinite in size, then the conventional thermal noise has the following form, derived by BHV [their Eq. (14) with $w_{o}=\sqrt{2} r_{o}$, which differs from the formula derived earlier by Levin [6]—-his Eq. (2)]:

$$
S_{q}^{\mathrm{ITM}}=\frac{4 k_{\mathrm{B}} T}{\omega} \frac{1-\sigma^{2}}{\sqrt{2 \pi E r_{o}}} \Phi(\omega)
$$

As for thermoelastic noise, we define a finite-test-mass correction $C_{\text {FTM }}^{2}$ to be the ratio of the finite-test-mass spectral density (58) to that (59) for the infinite test mass:

$$
C_{\mathrm{FTM}}^{2}=\frac{S_{q}^{\mathrm{FTM}}}{S_{q}^{\mathrm{ITM}}} .
$$

We plot the square root of this correction (i.e., the amplitudenoise correction) as a function of beam-spot radius $r_{o}$ in Fig. 3 for a LIGO-II type test mass. We show there two curves, $C_{\text {FTM }}$ as given by the BHV formulas, and as given by our 


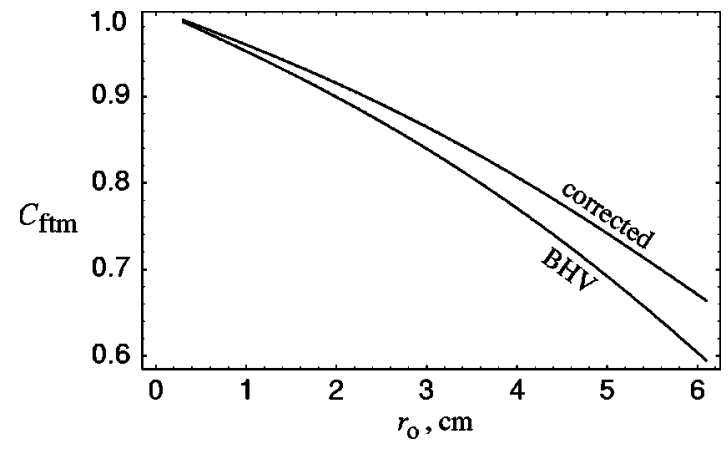

FIG. 3. $C_{\mathrm{FTM}}=\sqrt{S_{q}^{\mathrm{FTM}}} / \sqrt{S_{q}^{\mathrm{ITM}}}$, the finite-test-mass correction to the conventional, homogeneous thermal noise, as a function of the beam-spot radius $r_{o}$, for test masses with the parameters currently being contemplated for LIGO-II: $30 \mathrm{~kg}$ sapphire cylinders with radius $a=14 \mathrm{~cm}$ and thickness $H=12.2 \mathrm{~cm}$. The curve labeled "BHV" is the result derived in Ref. [5] [their Eqs. (29), (28), and equation following (31)]; the curve labeled "corrected" is our corrected version of their result [our Eqs. (58), (52) and (54)].

corrected formulas. Note that the BHV errors have only a small influence: their noise was too low by a factor $\lesssim 5$ percent when $r_{o} \lesssim 4 \mathrm{~cm}$.

\section{CONCLUSION}

In this paper we have sketched a fairly simple method of analyzing thermoelastic thermal noise in interferometric detectors, we have used that method to derive formulas for the noise in cylindrical test masses with finite radius, thickness, and beam spots, and we have corrected the corresponding finite test-mass formulas for conventional thermal noise. Our formulas should be useful in optimizing the test-mass designs for interferometric gravitational wave detectors.

Because thermoelastic noise arises from physical processes associated with ordinary thermal fluctuations, thermal conductivity and thermal expansion, and is not influenced by " dirty" processes such as lattice defects and impurities (except through the easily measured conductivity and expansion), the predictions for thermoelastic noise should be very reliable. Nevertheless, experimental tests of the theory would be useful and are being planned.

Other forms of thermal noise do rely in crucial, illunderstood ways on dirty processes and thus are far less reliably understood than thermoelastic noise. This is especially the case of thermal noise associated with (inhomogeneous) dissipation in and just beneath the test mass's dielectric-mirror coatings [for which Levin [6] predicts, in the infinite-test-mass limit, a dependence $S_{q} \propto 1 / r_{o}^{2}$ on beam- spot radius, compared to $S_{q} \propto 1 / r_{o}^{3}$ for thermoelastic noise and $S_{q} \propto 1 / r_{o}$ for conventional, homogeneous thermal noise]. Detailed experimental studies of these other forms of thermal noise are much needed as part of the research and development for interferometric gravitational-wave detectors, and are being planned.

In some of the planned experiments, very small beam radii $r_{o}$ and/or high frequencies $f$ may be used. For

$$
\begin{aligned}
r_{o} \lesssim r_{o}^{\text {heat }} & \equiv \sqrt{\frac{\kappa}{C_{V} \rho f}} \\
& \simeq 0.4 \mathrm{~mm} \sqrt{\frac{100 \mathrm{~Hz}}{f}} \text { for sapphire, }
\end{aligned}
$$

the adiabatic approximation breaks down seriously [cf. Eq. (9) and associated discussion] and our analysis of thermoelastic noise must be redone taking account of the diffusive redistribution of temperature during the elastic oscillations. Some foundations for doing this have been laid by $\mathrm{BGV}$ [4]. For frequencies

$$
\begin{aligned}
f \gtrsim f_{\text {sound }} & \equiv \frac{c_{s}}{\min (a, H)} \\
& \simeq 10^{4} \mathrm{~Hz} \frac{10 \mathrm{~cm}}{\min (a, H)} \quad \text { for sapphire }
\end{aligned}
$$

(where $c_{s}$ is the sound speed), the quasistatic approximation breaks down seriously [cf. Eq. (6) and associated discussion], and our analysis must be redone taking account of the finite propagation speed of the test mass's elastic deformations.

After completing our analysis of thermoelastic noise in finite sized test masses, we learned that Sergey Vyatchanin [9] has been carrying out an analysis of this same issue, but using somewhat different techniques. In writing the final version of this paper, we have benefitted from email exchanges with him.

\section{ACKNOWLEDGMENTS}

For helpful advice we thank Michael Gorodetsky, Eric Gustafson, James Hough, David Shoemaker, Jean-Yves Vinet, Rainer Weiss, and especially Vladimir Braginsky and Sergey Vyatchanin. A lively interchange of email with Vyatchanin has helped us understand more deeply the issues underlying thermoelastic noise, and we expect that his manuscript [9] will shed valuable new light on those issues. This research was supported in part by National Science Foundation Grant PHY-9900776.
[1] P. R. Saulson, Fundamentals of Interferometric Gravitational Wave Detectors (World Scientific, Singapore, 1994); see especially Chap. 7, and Fig. 16.2 and associated discussion.

[2] H. B. Callen and T. A. Welton, Phys. Rev. 83, 34 (1951).

[3] B. C. Barish and R. Weiss, Phys. Today 52, 44 (1999); see also http://www.ligo.caltech.edu/
[4] V. B. Braginsky, M. L. Gorodetsky, and S. P. Vyatchanin, Phys. Lett. A 264, 1 (1999); cited in text as BGV. In this paper, thermoelastic noise is referred to as noise due to thermodynamical fluctuations, because it arises when thermodynamical fluctuations of the distribution of heat inside the test mass produce, via the finite thermal expansion coefficient, 
changes of the test-mass shape. BGV refer to homogeneous thermal noise as due to Brownian fluctuations because it is caused by a Brownian-motion-type feeding of thermal energy into and out of the test mass's low-order normal modes of vibration.

[5] F. Bondu, P. Hello, and J.-Y. Vinet, Phys. Lett. A 246, 227 (1998); cited in text as BHV. This paper contains a number of errors, mostly typographical. Because the paper is so fundamental to thermal-noise modeling, the following list of errors may be of help to other researchers. [Vinet (private communication) agrees with this list.] In Eqs. (3) and (5) the denominator $\mu$ should be $\lambda+\mu$ [same as in Eqs. (4) and (6)]; assuming the Landau-Lifshitz sign convention for the stress tensor as in Appendix A of BHV, the second boundary condition after Eq. (8) should be $\Theta_{z z}(r, z=0)=-p(r)$ and the last boundary condition after Eq. (17) should be $\Theta_{z z}(r, z=0)=-p(r)$; the overall minus signs in Eqs. (9)-(11) should all be changed to + ; there should be a factor $\mu$ in the denominator of Eq. (12); in the equation for $p_{m}$, preceding Eq. (19), the $\sigma$ in the de- nominator should be $\pi$; in Eq. (19), in the third term on the right side, $k_{m} a$ should be $k_{m} z$ [cf. our Eq. (33)]; Eqs. (21)(24) should take the forms given in our Eqs. (35a)-(35e); and Eqs. (28) and (31) for $\Delta U$ should take the forms given in our Eqs. (54), (55) and (57).

[6] Yu. Levin, Phys. Rev. D 57, 659 (1998). Note that Levin made an error in calculating the oscillating elastic energy, when applying his method to conventional, homogeneous thermal noise. His Eqs. (A5) and (14) should actually have the form derived by BHV [5] [their Eq. (13)], and correspondingly, his final formula (1), (15) for the conventional thermal noise should actually be that derived by BHV [their Eq. (14) with $w_{o}=\sqrt{2} r_{o}$; our Eq. (59)].

[7] L. D. Landau and E. M. Lifshitz, Theory of Elasticity, third ed. (Pergamon, Oxford, 1986).

[8] A. E. H. Love, A Treatise on the Mathematical Theory of Elasticity, fourth ed. (Cambridge University Press, Cambridge, England, 1927; reprinted by Dover, New York, 1944).

[9] S. P. Vyatchanin (in preparation). 